\title{
Revue de psychoéducation
}

Meilleur, I., Proulx, A., Bachelet, T., et Arsenault, A. (2016). Au-delà des mots. Le trouble du langage chez l'enfant. Montréal, Québec, Éditions du CHU Sainte-Justine

\section{Carole Sénéchal}

Volume 46, numéro 1, 2017

URI : https://id.erudit.org/iderudit/1039693ar

DOI : https://doi.org/10.7202/1039693ar

Aller au sommaire du numéro

Éditeur(s)

Revue de Psychoéducation

ISSN

1713-1782 (imprimé)

2371-6053 (numérique)

Découvrir la revue

Citer ce compte rendu

Sénéchal, C. (2017). Compte rendu de [Meilleur, I., Proulx, A., Bachelet, T., et Arsenault, A. (2016). Au-delà des mots. Le trouble du langage chez l'enfant. Montréal, Québec, Éditions du CHU Sainte-Justine]. Revue de psychoéducation, 46(1), 248-249. https://doi.org/10.7202/1039693ar

Tous droits réservés @ La Corporation de la Revue Canadienne de Psycho-Éducation, 2017
Ce document est protégé par la loi sur le droit d'auteur. L'utilisation des services d’Érudit (y compris la reproduction) est assujettie à sa politique d'utilisation que vous pouvez consulter en ligne.

https://apropos.erudit.org/fr/usagers/politique-dutilisation/ 


\section{- Meilleur, I., Proulx, A., Bachelet, T., et Arsenault, A. (2016). Au-delà des mots. Le trouble du langage chez l'enfant. Montréal, Québec, Éditions du CHU Sainte-Justine.}

Dysphasie, trouble spécifique de langage, trouble primaire de langage: les termes varient, la réalité est une. Certains enfants éprouvent des difficultés persistantes d'acquisition du langage, lorsque le retard " est significatif et qu'il persiste sans raison évidente, c'est-à-dire que l'enfant est encouragé à parler, qu'il souhaite interagir, que son audition est suffisante pour développer le langage et que sa motricité de même que son développement intellectuel ne peuvent être mis en cause » (p. 19).

Au-delà de la grande variabilité des portraits cliniques, il s'agit principalement de difficultés au niveau de la prononciation, de l'acquisition des mots, de l'élaboration des phrases, de la communication et du langage écrit. Les défis que pose ce problème sont amplifiés par la présence de plusieurs autres troubles ou encore par des difficultés de comportement qui peuvent résulter des déficits langagiers.

Le livre est structuré en 3 parties. La première partie, qui campe cinq chapitres, aide à comprendre le trouble du langage chez l'enfant et à en cerner les spécificités parmi d'autres troubles de développement associés (p.ex. le TDAH, des difficultés motrices, la fragilité sensorielle, l'anxiété) et plusieurs problèmes de l'enfance présentant des traits communs (p.ex. trouble du spectre de l'autisme, retard global de développement, difficultés psychoaffectives).

La seconde partie comprend les chapitres 6 à 11 et présente des stratégies concrètes aux familles pour stimuler au quotidien le développement du langage chez l'enfant dysphasique. En plus, des équipes interdisciplinaires, la famille offre « un milieu beaucoup plus riche en émotions, en affectivité et en expériences que la clinique ou le centre de réadaptation » (p. 107). Au-delà de l'évaluation et du diagnostic, la vie quotidienne est riche en occasions d'interaction, de rétroaction et de renforcement naturel qu'il faut savoir exploiter pour soutenir la communication des enfants dysphasiques, que ce soit à travers les jeux, la lecture ou les routines quotidiennes. Des stratégies ciblant spécifiquement l'acquisition du langage (p.ex. ralentissement de la parole, modulation accentuée de la voix, phrases courtes, pauses et reformulations) sont certes nécessaires. Toutefois, les interventions doivent aussi tenir compte de la répercussion possible des difficultés de compréhension et/ ou d'expression sur le comportement de l'enfant, pour mieux en identifier l'origine du problème et trouver des solutions adaptées sur le plan notamment de la gestion des émotions (frustrations, perte de motivation, manifestations d'opposition, résistance aux changements), des difficultés relationnelles ou du cheminement scolaire.

La troisième partie (chapitre 12) se base principalement sur les études menées pendant plusieurs années au Royaume-Uni, aux États-Unis et au Canada pour aborder les enjeux psychologiques et sociaux, incluant les préoccupations et les vulnérabilités qui risquent de perdurer chez les enfants dysphasiques devenus 
adultes, et ce, dans différentes sphères de leur vie, dont la réussite scolaire, la vie sociale, la vie amoureuse, l'acquisition de l'autonomie et l'accès au monde du travail.

Inspiré par les contacts privilégiés au quotidien avec des enfants présentant un trouble de langage et leur entourage, les auteures ont rédigé un ouvrage dans le but avoué de « fournir une réponse éclairée et globale aux questions pertinentes et stimulantes soulevées par les parents " d'enfants dysphasiques. Les auteures Isabelle Meilleur (orthophoniste), Annick Proulx (travailleuse sociale), Tamara Bachelet (neuropsychologue) et Annik Arsenault (ergothérapeute) œuvrent en effet depuis plusieurs années dans le milieu de la réadaptation auprès d'une clientèle de jeunes présentant un déficit du langage.

C'est dans cette intime connaissance du vécu et de l'expérience des enfants et de leur famille que réside le point fort de l'ouvrage. Les capsules récapitulatives reprennent l'essentiel de chaque chapitre en concluant avec des points importants " à retenir ". Au fil des pages, les explications sont ponctuées de mises en situation et d'exemples évocateurs pour en faciliter la compréhension. Sans remplacer une évaluation formelle par des professionnels compétents, ces illustrations au cas par cas aident le lecteur peu familiarisé avec les troubles de développement de l'enfance à mieux en "visualiser ॥ les enjeux, quand vient le temps de départager entre un trouble persistant de langage et un retard passager (p. 24) ou encore différentes difficultés pouvant coexister avec le trouble du langage ( $p .72$ et 85 ).

Ces mises en situation sont d'autant bienvenues dans la deuxième partie de l'ouvrage au cours de laquelle les auteures invitent les familles à s'impliquer dans le processus d'intervention. Pour ce faire, elles présentent différentes stratégies pour stimuler le développement du langage chez l'enfant dysphasique. Au-delà des recommandations conceptuelles, telles « enrichir le langage par petites touches » (p. 127) ou « faire parler l'enfant sans le faire répéter » (p. 144). À cet effet, les auteures ont pris soin d'en illustrer l'application concrète à l'aide d'exemples tirés du quotidien, en exhortant par exemple les parents à reformuler les phrases de l'enfant, à en accentuer les éléments manquants (p. 127). De plus, quelques façons d'inciter l'enfant à parler davantage sont présentées dans un tableau qui associe chaque conseil à un exemple de dialogues (p. 144).

Une autre qualité de l'ouvrage consiste dans sa préoccupation de situer le trouble du langage parmi d'autres troubles de développement présentant des traits communs et d'aborder différents problèmes associés qui résultent éventuellement des difficultés de compréhension/expression. Rattacher ces difficultés concomitantes à une origine commune peut aider grandement les parents soucieux d'accompagner au quotidien leurs enfants présentant des déficits langagiers.

Si le livre s'adresse principalement aux parents, tous ceux et celles qui s'intéressent de près ou de loin aux enfants qui présentent un trouble du langage, y trouveront des réponses à certaines de leurs questions ainsi que des références thématiques à la fin des chapitres. 